\title{
ELEMENT ABUNDANCE RATIOS IN GALACTIC BULGE STARS
}

\author{
R. MICHAEL RICH \\ Department of Astronomy, Columbia University \\ Mail Code 5242, New York, NY, 10027
}

I review the current status of measurements of element ratios in metal rich stars. These include giants in the Galactic bulge, metal rich Galactic globular clusters, and high velocity dwarfs in the Solar vicinity. There is evidence for enhancement of $\mathrm{Mg}$ and $\mathrm{Ti}$ in the bulge. While s-process elements have Solar abundance in bulge giants, they are deficient in the metal rich, high velocity dwarfs. There are no secure measurements of oxygen abundance in the bulge, but present data indicate no evidence either for extremely low or high [O/Fe] in bulge giants. The current data are limited by resolution and $S / N$, and I outline goals for observations with the next generation of ground-based telescopes.

\section{Introduction}

If the central bulge of the Milky Way formed rapidly, one would expect that enrichment could occur only through massive star SNe, with little or no contribution from core deflagration SNe. In this scenario, one expects alpha-capture elements to be enhanced at a given $[\mathrm{Fe} / \mathrm{H}]$ compared to the Solar vicinity: element abundance ratios may record something of the history of star formation. The idea is developed in Wheeler, Sneden \& Truran (1989) and its application to bulges and elliptical galaxies is developed in Matteucci \& Brocato (1990). As Kraft reviews in this volume, the stellar evolution effects that modify surface abundances in globular cluster stars do not appear to be a problem for field giants.

The Galaxy actrally has a number of metal rich stellar populations, and their evolutionary histories vary greatly and properties of these stars may be quite different. There are the well known giants in the central bulge of the Galaxy, particularly well observed in Baade's Window $\left(l=-4^{\circ}, b=-8^{\circ}\right)$. There are also a substantial number of globular clusters at high metallicity in the Galactic Bulge and disk (Ortolani et al. 1995) and metal rich open clusters in the disk (Phelps \& Janes 1996). Grenon (1989) has identified a group of high velocity, metal rich dwarfs in the disk, and he suggests that they may have originated in the bulge. Needless to say, there is much effort needed on a systematic study of element ratios in all of these populations. Feltzing (also in this volume) reviews the growing body of data on metal rich stars in the disk. Further details are found in the review of McWilliam (1997).

\section{The Galactic Bulge}

\section{1. $[\mathrm{FE} / \mathrm{H}]$}

Because of the distance and reddening, it has been difficult to establish the abundances of the bulge giants. The first low resolution efforts by Whitford \& Rich (1983) and Rich (1988) found that the bulge giants were in general metal rich, with a wide range in metal abundance. The Rich (1988) scale found a median [Fe/H] of twice Solar, but the abundances were based on the sum of the $\mathrm{Fe}$ and $\mathrm{Mg}_{2}$ Faber indices. Only with higher resolution, higher $\mathrm{S} / \mathrm{N}$ data, it has been possible to approach the true $[\mathrm{Fe} / \mathrm{H}]$ scale, which has a median of -0.15 dex (McWilliam \& Rich, 1994; MR94). However, there remain some concerns: Geisler \& Friel (1992) confirmed the Rich (1988) scale with Washington photometry. Further, given that light elements in the disk are known to have an abundance gradient, it is hard to understand how the bulge could be more metal poor than the Solar neighborhood. There is reason to be concerned that at the $R=17,000$ of the MR94 study, the derived continuum may have been erroneously low due to unresolved iron lines, and the 
iron abundance may actually be much higher, in agreement with the low resolution analysis (where total equivalent widths of the strong lines might actually be easier to measure). There are 2 recent studies that strengthen confidence in the Fe abundance scale. Castro et al. (1996) analyzed Keck Hires spectra ( $R=38,000 ; S / N=30$ ) of the metal rich disk giant $\mu$ Leo and the bulge giant $B W$ IV167. Due to incomplete wavelength coverage and low $S / N$, it was possible to only measure the iron abundance. However, the bulge giant and $\mu$ Leo are indistinguishable, with $[\mathrm{Fe} / \mathrm{H}]=+0.47 \pm 0.03$ dex. Their results are in excellent agreement with MR94's analysis of the same star and one may therefore conclude that doubling the resolution did not change the result. Peterson \& Green (1997) have analyzed a hot horizontal branch star member of the metal rich old open cluster NGC 6791 . Hot enough so that difficult sources of blanketing (such as CN) are not a problem, the analysis finds $[\mathrm{Fe} / \mathrm{H}]=+0.3-0.4 \mathrm{dex}$, with $[\alpha / \mathrm{Fe}]$ enhanced by $+0.2 \mathrm{dex}$. They also find that the cool giants in the cluster are indistinguishable from $\mu$ Leo.

At the present time, then, the iron abundance scale looks reasonably secure. The possibility that the continuum is blanketed by weak Fe I lines can be largely ruled by these 2 studies. MR94 also found that $\mathrm{CN}$ lines could have the pernicious effect of causing a spuriously high Fe abundance, as many of the vibration/rotation lines coincide with Fe I lines at low resolution. Again, these two studies serve to confirm that the MR94 analysis (and their list of clean lines) does not suffer from this problem. It would be useful to use 8 and $10 \mathrm{~m}$ class telescopes to obtain echelle spectra of hot HB stars in metal rich Galactic Center globular clusters such as NGC 6553 and 6528 and to compare giants in these clusters with those of the bulge.

\subsection{ALPHA-CAPTURE ELEMENTS}

One would most like to know if oxygen is enhanced in the bulge, as it is the most abundant alphacapture element produced in the hydrostatic shells of massive pre-supernova stars. Unfortunately, the single usable metastable line at $6300.3 \AA$ suffers from blending with an Sc II line at 6300.7 and with numerous weak telluric features in the regions. For stars of low radial velocity, the night sky emission line cannot be accurately subtracted, and the feature is lost. MR94 found no evidence for any dramatic oxygen enhancement, a result confirmed in the more careful effort of McWilliam, Rich, \& Tomaney (1998).

As discussed in MR94, magnesium abundances are especially hard to measure; few $g f$ values are available, and some of the $\mathrm{Mg}$ lines give systematically discrepant abundances. The MR94 abundances were typically based on spectrum synthesis of 3 lines per star. Approximately 4 to 6 lines of $\mathrm{Ca}$ and $\mathrm{Si}$, respectively, were usable by MR94, although some of these lines are uncomfortably strong in metal rich stars. With its good representation of many medium strength lines, Ti I was well measured in the MR94 stars.

MR94 find a puzzling enrichment pattern for the alpha-capture elements, in which $\mathrm{Mg}$ and $\mathrm{Ti}$ approximately follow the expected enhancement, but $\mathrm{Si}$ and $\mathrm{Ca}$ follow the Solar neighborhood disk enrichment line (Fig. 1). MR94 compared the measured line strengths at constant $T_{\text {eff }}$ for Ti lines in the bulge giants and in a large sample of $671 \mathrm{~K}$ giants (McWilliam 1990) and the enhancement of $\mathrm{Ti}$ in the bulge giants is clear. Calculated yields from Type II SN models covering a wide range of parameter space do not produce ejecta in which $\mathrm{Mg}$ and $\mathrm{Ti}$ are selectively enhanced relative to $\mathrm{Ca}$ and Si (Nomoto et al. 1997).

Low resolution studies by Terndrup et al. (1995) and Sadler et al. (1996) argue that Mg must be enhanced in the majority of bulge giants, and the Mg enhancement was introduced by MR.4 to explain the discrepant R88 abundance scale. There is always the risk with these low resolution studies (as is the case for the giant ellipticals) that they confirm the expected result. The element ratios will be considered secure in the bulge when they have been determined at high resolution and high $\mathbf{S} / \mathrm{N}$ for a larger sample.

\subsection{HEAVY ELEMENTS}

Because s-process elements are produced in the envelopes of AGB stars, their presence indicates that the population had an enrichment timescale of at least $10^{8} \mathrm{yr}$; in the extreme metal poor halo, s-process is very deficient; MR94 find Solar abundances for La, Y, and Ba in the bulge giants. The $r$-process is associated with massive star SNe, and the abundance analysis of the peculiar extreme 
metal poor star CS22892-052 (Sneden et al. 1996) supports this association. Eu was measured in most of the bulge giants by MR94, and found to be mildly enhanced.

\subsection{LIGHT ELEMENTS}

MR94 find one super Li-rich giant, a curiosity since the canonical theories explaining such an enhancement (e.g. Scalo, Despain, \& Ulrich 1975) require evolved luminous stars on the AGB, presumably the progeny of stars more massive than the Galactic bulge is known to have (Ortolani et al. 1995). High $\mathrm{Na}$ and $\mathrm{Al}$ abundances in the bulge giants are most likely related to the abundance anomalies seen in globular clusters giants (see $\mathrm{Kraft}$ in this volume). If present, such anomalies may raise a red flag concerning the alpha-capture elements as well although this is still not considered to be a problem for giants in field populations.

\section{Relating the Bulge to Other Populations}

It would be interesting to know if any other populations share the chemical evolution history of the bulge, or if there are any bulge stars that migrate through the Solar neighbhorhood. Given the similarity in colors between bulges and inner disks established from photometic surveys (Balcells \& Peletier 199X), could the inner disk and bulge share a similar detailed chemical history?

The most obvious population to start with are the globular clusters in the bulge whose spatial and abundance distributions are now known to be very similar to that of the bulge field stars (Bica, Barbuy, \& Ortolani, 1997). The only published high resolution study (Barbuy et al. 1992) finds $[\mathrm{M} / \mathrm{H}]=-0.2 ;$ Barbuy's continuing analysis of this cluster suggests a slightly lower metal abundance and modest +0.3 dex enhancements in $\mathrm{Mg}_{\mathrm{g}}$ and $\mathrm{Ti}$.

Grenon (1989) has identified metal rich stars in the Solar Neighbhorhood with high eccentricity orbits, and he suggests they are local interlopers from the bulge. This idea has gained some momentum with the discovery of the bar-like nature of the bulge, which might allow some stars on chaotic orbits to be ejected. Barbuy \& Grenon (1990) found enhanced oxygen in these high velocity stars, appearing to strengthen their connection to the bulge. Two studies of these metal rich disk stars have recently appeared; the results now make a bulge connection less likely. Castro et al. (1997) analyzes a small sample of high metallicity, high velocity dwarfs from Grenon's sample. She finds that $[\mathrm{O} / \mathrm{Fe}]$ declines with $[\mathrm{Fe} / \mathrm{H}]$, falling below the Solar value in metal rich stars. $\mathrm{Mg}$ and $\mathrm{Si}$ follow iron, while $\mathrm{Ca}$ and $\mathrm{Ti}$ decline like oxygen. In contrast to the bulge, the s-process elements are subsolar $(-0.5$ dex). These results are largely confirmed (but the alpha-capture elements follow iron) in the larger sample of Feltzing \& Gustafsson (1998), which is not a kinematically selected sample. In contrast to Edvardsson et al. 1993, Feltzin \& Gustafsson find no evidence for correlations between abundances and kinematics.

As far as $\mathrm{Mg}$ and $\mathrm{Ti}$ are concerned, one cannot rule out common chemical evolution for the Castro et al. disk stars and the bulge giants. The star G161-29 also has a strong Eu line. However, the 0.5-1.0 dex difference in s-process between the bulge and disk stars remains as a considerable obstacle to their association.

As a footnote, I mention the work of Sellgren et al. (1997) on Galactic center supergiants (she gives a full report in the proceedings of IAU Symposium 187). She finds $[\mathrm{Fe} / \mathrm{H}] \approx \mathrm{Solar}$ for 11 stars, but the stars in question have formed only in the last $10^{7} \mathrm{yr}$, and probably more reflect bar-driven gas inflow rather than the chemical evolution of the bulge.

\section{Discussion}

Although the bulge may have formed early and quickly, such a history is not reflected in dramatic enhancements of the alpha-capture elements thought to be produced in massive star SNe. It would be interesting to isolate the most metal poor stars in the bulge and to compare their detailed compositions with extreme stars of the outer halo. If the bulge formed along with the very first stellar generation, the s-process elements should be deficient in the most metal poor stars. If the material that formed the bulge was processed in some prior generation of stars (e.g. in the halo) then one might expect the s-process elements to tend toward Solar. In fact, the low s-process in the metal rich disk stars is puzzling, since their tendancy to have lower oxygen at higher $[\mathrm{Fe} / \mathrm{H}]$ 
follows the Solar vicinity enrichment line and would be consistent with a slower enrichment (hence less ancient composition).

In further considering the history of the bulge, the work of Ortolani et al. (1995) leaves little doubt that the field population of the bulge 500 pc distant from the nucleus (Baade's Window) is as old as the metal rich globular clusters. There is also strong (and ever accumulating) evidence for the bar-shaped morphology of the bulge field population. However, theoretical simulations of bars suggest that they do not survive for a Hubble Time. The chemistry does not provide a strong constraint, except to rule out the most extreme starburst scenarios. There is clearly great need for more spectroscopy of bulge giants at a range of galactocentric distance. The Galactic center has a more complex star formation history, and detailed abundance analysis of stars representing the range of population ages will be of great interest in the coming years. Finally, the abundances of the old disk stars and old open clusters such as NGC 6791 are of great interest in that they illustrate that stellar populations can attain high metallicity in the disk, at lower density and larger galactocentric distance than one usually associates with metal rich populations. The starburst events that lead to high metallicity may depend more on the local environment than on the global environment, or the initial mass function might vary with time. Both NGC 6791 and the Grenon sample are $\approx 10^{10} \mathrm{yr}$ old, and it is not clear why the ISM could reach such high metallicities early on given that the ISM is not so metal rich today. Such issues lay clear the importance of having a dedicated campaign to measure abundance ratios using the next generation of large telescopes.

\section{References}

Barbuy, B., Castro, S., Ortolani, S., \& Bica, E. 1992, A \&A, 259, 607

Barbuy, B., Ortolani, S., Bica, E., \& Renzini, A., Guarnieri, M.D. 1997,IAU Symp. 187, 203

Barbuy, B., Bica, E., \& Ortolani, S. 1997. A\&A, in press

Castro, S., Rich, R.M., McWilliam, A., Ho, L.C., Spinrad, H., Filippenko, A.V., \& Bell, R.A. 1996, AJ, 96, 1319

Castro, S., Rich, R.M., Grenon, M., Barbuy, B., \& McCarthy, J.K. 1997, AJ, 114, 376

Geisler, D., \& Friel, E.D. 1992, $A J, 104,128$

Grenon, M. 1989, Astrophys. Space Sci., 156, 29

Janes, K.A., \& Phelps, R.J., AJ, 111, 1604

Matteucci, F., \& Brocato, E. 1990, ApJ, 365, 539

McWilliam, A., 1990, ApJS, 74, 1075

McWilliam, A. 1997, ARAA, 35, 000

McWilliam, A., Rich, R.M. 1994, ApJS, 01, 749 (MR94)

McWilliam, A., Tomaney, A., \& Rich, R.M. 1998, in preparation

Nomoto, K., et al. 1997b, NuPhA. 616, 79

Ortolani, S., et al. 1995, Nat, 377, 701

Peletier, R.F., \& Balcells, M. 1996, AJ, 111, 2238

Peterson, R., \& Green, E.M. 1997, in preparation

Rich, R.M. 1988, $A J$, 95, 828

Sadler, E.M., Rich, R.M., \& Terndrup, D.M. 1996, $A J, 112,171$

Scalo, J.M., Despain, K.H., \& Ulrich, R.K. 1975, ApJ, 196, 805

Sellgren, K. et al. 1997, in preparation

Sneden, C., McWilliam, A., et al. 1996, ApJ, 467, 819

Terndrup, D.M., Sadlex, E.M., \& Rich, R.M. 1995, JA, 110, 1774

Wheeler, J.C., Sneden, C., \& Truran, J.W., Jr. 1989, ARAA, 27, 279

Whitford, A.E., \& Rich, R.M. 1983, ApJ, 274, 723 